\title{
ADD3 Deletion in Glioblastoma Predicts Disease Status and Survival
}

\author{
Karrie Mei-Yee Kiang, Stella Sun and Gilberto Ka-Kit Leung * \\ Department of Surgery, Li Ka Shing Faculty of Medicine, The University of Hong Kong, Queen Mary Hospital, Hong Kong, \\ Hong Kong SAR, China
}

\section{OPEN ACCESS}

Edited by:

Jose Javier Otero,

The Ohio State University,

United States

Reviewed by:

Andrei Ivanov,

Cleveland Clinic, United States

Teresa San-Miguel,

University of Valencia, Spain

*Correspondence:

Gillberto Ka-Kit Leung gillberto@hku.hk

Specialty section:

This article was submitted to Neuro-Oncology and

Neurosurgical Oncology,

a section of the journal

Frontiers in Oncology

Received: 31 May 2021 Accepted: 17 November 2021 Published: 14 December 2021

Citation:

Kiang KM-Y, Sun S and Leung GK-K (2021) ADD3 Deletion in Glioblastoma Predicts Disease Status and Survival.

Front. Oncol. 11:717793. doi: 10.3389/fonc.2021.717793
Loss of heterozygosity $(\mathrm{LOH})$ on chromosome 10 frequently occurs in gliomas. Whereas genetic loci with allelic deletion often implicate tumor suppressor genes, a putative tumor suppressor Adducin3 (ADD3) mapped to chromosome 10q25.2 was found to be preferentially downregulated in high-grade gliomas compared with low-grade lesions. In this study, we unveil how the assessment of $A D D 3$ deletion provides clinical significance in glioblastoma (GBM). By deletion mapping, we assessed the frequency of $\mathrm{LOH}$ in forty-three glioma specimens using five microsatellite markers spanning chromosome 10q23-10qter. Data were validated in The Cancer Genome Atlas (TCGA) cohort with 203 GBM patients. We found that allelic loss in both D10S173 (ADD3/MXI1 locus) and D10S1137 (MGMT locus) were positively associated with tumor grading and proliferative index (MIB-1). However, LOH events at only the ADD3/MXI1 locus provided prognostic significance with a marked reduction in patient survival and appeared to have diagnostic potential in differentiating high-grade gliomas from low-grade ones. Furthermore, we showed progressive loss of $A D D 3$ in six out of seven patient-paired gliomas with malignant progression, as well as in recurrent GBMs. These findings suggest the significance of ADD3/MXI1 locus as a promising marker that can be used to refine the $\mathrm{LOH} 10 \mathrm{q}$ assessment. Data further suggest the role of $A D D 3$ as a novel tumor suppressor, whereby the loss of $A D D 3$ is indicative of a progressive disease that may at least partially account for rapid disease progression in GBM. This study revealed for the first time the downregulation of $A D D 3$ on the genetic level resulting from copy number deletion.

Keywords: loss of heterozygosity, glioblastoma, deletion mapping, tumor suppressor, ADD3

\section{INTRODUCTION}

Glioblastoma (GBM), the most malignant primary brain tumor in adults, is often characterized by high levels of genomic instability. Loss of heterozygosity (LOH) of chromosome 10q is among the most frequent genetic alterations that occur in over $80 \%$ of de novo cases and $60 \%-70 \%$ of secondary cases, but it is less frequent in anaplastic astrocytoma (40\%) and rare in low-grade gliomas (1-4). Other frequent abnormalities in primary GBM include EGFR amplification (36\%), p16 ${ }^{I N K 4 a}$ deletion (31\%), TP53 mutation (28\%), and PTEN mutation $(25 \%)(5,6)$. The assessment of LOH10q genotype is not included in the recent WHO classification for gliomas (WHO 2016), as it is 
believed that the assessment of both chromosome $7+/ 10 q-$ and TERTp mutations is perhaps more informative than assessing EGFR amplification for the diagnosis of GBM (7). Moreover, most studies to date have identified $\mathrm{LOH}$ of $10 \mathrm{q}$ as a poor prognostic marker for high-grade gliomas (8-10). Allelic deletion on chromosome $10 \mathrm{q}$ has also been observed in various advanced human malignancies (11-14). This suggests that such allelic regions might harbor tumor suppressor genes and be involved in the development of cancer.

Allelic deletions can occur in the entire copy or part of chromosome 10. Several commonly deleted loci have been identified on chromosome $10 \mathrm{q}$ in the region spanning 10q23 to 10 qter, encompassing the allelic loci of a number of genes with well-established tumor-suppressive roles (e.g., MMAC/PTEN, $S U F U, F G F R 2$, and DMBT1) or putative tumor-suppressive roles (e.g., ADD3/MXI1, LGI1, and BTRC) (9, 15-17). When comparing cohorts with primary and secondary gliomas, the acquisition of a malignant phenotype with marked proliferative activity was observed in a deletion mapping on 10q25-qter (18). This further suggests the importance of tumor suppressor genes in 10q25-qter apart from PTEN that are likely to be involved in the malignant progression of glioma.

Among these putative tumor suppressor genes, ADD3 was found to be downregulated in high-grade gliomas when compared with its less malignant counterpart in several gene expression profiling studies (19-21). ADD3 is located on chromosome 10q25.1-25.2, which is known to be a functional tumor suppressor region. While it primarily functions as a cytoskeleton protein, $A D D 3$ is characterized as a negative regulator of tumor growth and is negatively associated with malignant phenotypes such as angiogenesis in GBM (22). However, the basis behind $A D D 3$ downregulation has not been identified for which both genetic and epigenetic modifications should be taken into consideration when studying its functional characteristics in cancer cells. Moreover, although PTEN locus is often tested for chromosome LOH10q status, the frequency of allelic deletion on other $10 \mathrm{q}$ loci has not been adequately addressed in previous studies.

Here, we performed deletion mapping analysis in a glioma cohort to determine the regions of allelic loss in chromosome $10 \mathrm{q}$ and to establish correlations between their accumulation and different pathological phenotypes. By correlating patient characteristics to the occurrence of allelic loss, we determined whether the aberrant expression of $A D D 3$ in malignant glioma results from $\mathrm{LOH} 10 \mathrm{q}$, which may be involved in tumor relapse and malignant progression. We will also evaluate and highlight the potential application of $A D D 3$ as a novel biomarker for diagnostic, prognostic, and future therapeutic implications.

\section{MATERIALS AND METHODS}

\section{Human Glioma Tissue Specimens}

Fresh tumor tissues were snap-frozen in liquid nitrogen immediately after surgical resection at our institution. All specimens were obtained with informed consent from the patients. The study protocol was approved by our Institution Review Board. Diagnosis and histological classification were confirmed by specialists according to the WHO's brain tumor classification system before the release of the WHO 2016 classification on gliomas. Forty-three glioma specimens with WHO grades 2-4 (8 grade 2, 12 grade 3, and 23 GBM/grade 4) were collected for clinical and molecular analyses in the study. Specimens were obtained at the time of surgery between 2009 and 2016 and were stored at $-80^{\circ} \mathrm{C}$ until prior study use. All diagnoses were made radiologically via MRI before surgery and confirmed histologically by a certified pathologist. Tumors from another cohort of ten patients (P1-P10) who developed recurrence after the initial surgical treatment of the disease were also collected during the second operation for longitudinal comparison in our study.

\section{Clinical Data}

The records of forty-three patients were retrieved from their medical records from initial consultation to the latest follow-up. The data included the patient's age, gender, date of diagnosis, pathological diagnosis, initial tumor location, MRI-confirmed tumor size, and nature of the specimen types (primary or recurrent tumor). Other characteristics were $M G M T$ promoter methylation status, cycles of temozolomide treatment, survival time in days after treatment, and patient status at the last followup (dead or alive). Missing data and patients who were lost to follow-up were not counted in the study. The study cutoff date was November 30,2020, and patients who survived through this date were defined as the last follow-up date.

\section{PCR and PCR-Based Microsatellite Loss of Heterozygosity Analysis}

Genomic DNA was extracted from frozen tumor tissue using PureLink Genomic DNA mini kit (Life Technologies), according to the manufacturer's protocol. After DNA extraction, $50 \mathrm{ng}$ of genomic DNA was amplified using AmpliTaq gold 360 master mix (Applied Biosystems) by PCR. Reaction mix measuring $25 \mu \mathrm{l}$ was subjected to an initial denaturation cycle of $95^{\circ} \mathrm{C}$ for $7 \mathrm{~min}$, followed by 35 cycles of denaturation at $95^{\circ} \mathrm{C}$ for $30 \mathrm{~s}$, annealing at $58^{\circ} \mathrm{C}-62^{\circ} \mathrm{C}$ for $30 \mathrm{~s}$, and extension at $72^{\circ} \mathrm{C}$ for $30 \mathrm{~s}$, with a final extension at $72^{\circ} \mathrm{C}$ for $7 \mathrm{~min}$.

$\mathrm{LOH}$ on chromosome $10 \mathrm{q}$ was studied by PCR-based microsatellite analysis of the frequently deleted regions on chromosome 10q. Five microsatellite markers spanning across 10q23-qter were selected according to the genetic loci on the UCSC Table Browser on Human (GRCh37/hg19) assembly. Primer sequences were purchased from Life Technologies. Microsatellite marker D10S579 flanked PTEN gene (10q23.2); D10S198 is an intragenic marker within CNNM1 gene (10q24.2); D10S173 is intragenic within MXI1 and is $50 \mathrm{~kb}$ adjacent to ADD3 gene (10q25.2); D10S1483 is intragenic of FGFR2 gene (10q26.13); and D10S1137 flanked MGMT gene (10q26.3). The cytogenetic localization and the approximate genetic distances are shown in Figure 1. Tumor DNA samples were analyzed by capillary gel electrophoresis. DNA fragments from the PCR 


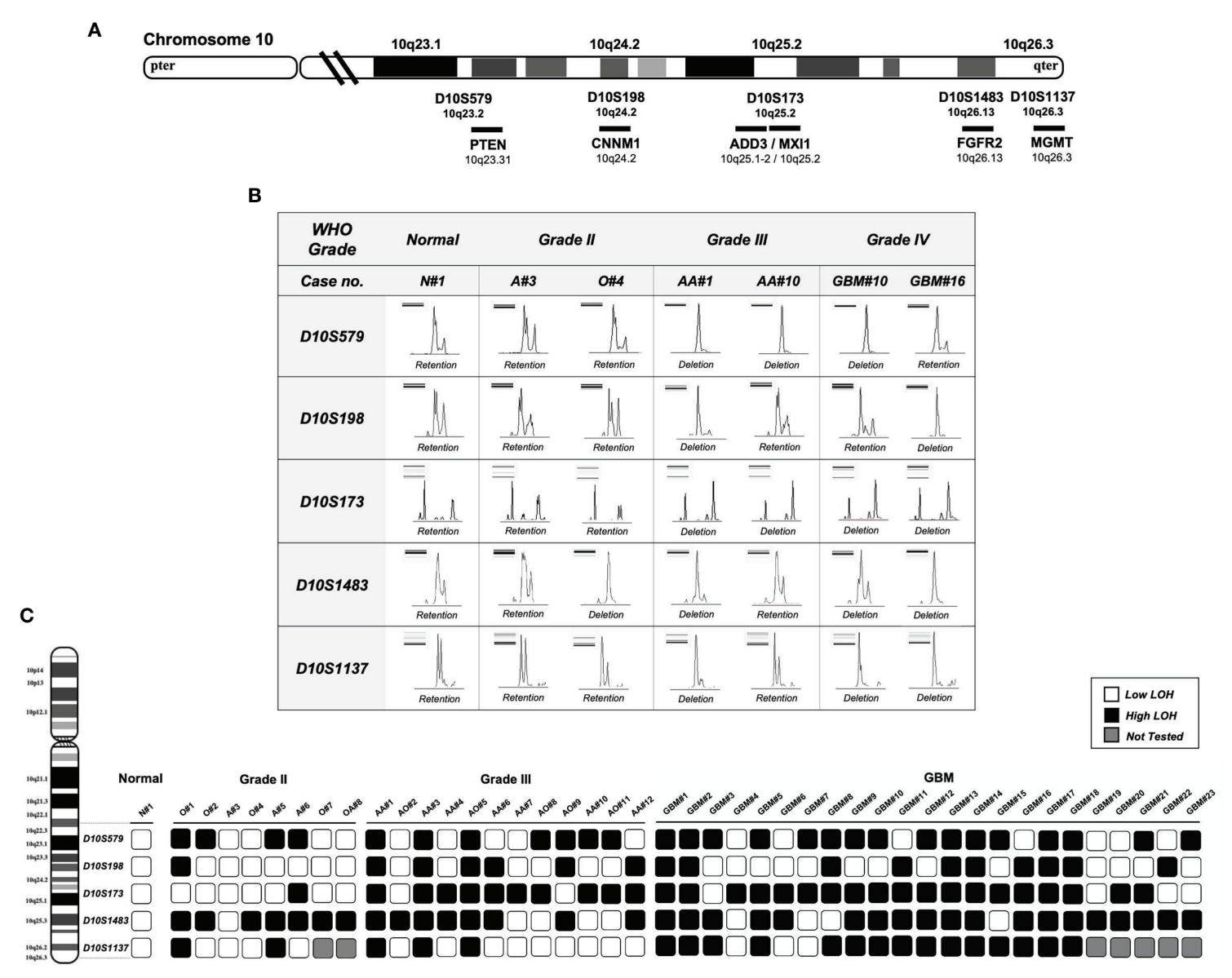

FIGURE 1 | Deletion mapping of chromosome 10q using PCR-based loss of heterozygosity (LOH) analysis. (A) The five polymorphic microsatellite markers from 10q23 to 10q26 (D10S579, D10S198, D10S173, D10S1483, and D10S1137) and the corresponding chromosomal loci flanking or intragenic of the gene locus (chromosome map based on UCSC GRCh37/hg19 assembly). (B) Assessment of allelic loss in fragment analysis on an automated capillary electrophoresis system (from seven representative cases). Allelic status was calculated based on the peak intensity ratios in gliomas relative to normal brain tissue. $\mathrm{LOH}$ ratio of $>0.5$ or $<1.5$ is defined as a low level of $\mathrm{LOH}$, and a ratio of $\leq 0.5$ or $\geq 1.5$ is defined as a high level of $\mathrm{LOH}$. DNA bands from gel electrophoresis are indicated on the top left corner of each panel. (C) Deletion map of 43 glioma specimens. Case numbers are indicated at the top of each column. Open and solid boxes represent low and high levels of $\mathrm{LOH}$, respectively. Boxes in gray were not tested. N, normal; $\mathrm{A}$, astrocytoma; O, oligodendroglioma; $\mathrm{AA}$, anaplastic astrocytoma; $\mathrm{AO}$, anaplastic oligodendroglioma; GBM, glioblastoma.

reaction were separated by a Fragment Analyzer automated CE system (Agilent). Allelic loss for each microsatellite locus was determined by the evaluation of peak intensity using PROSize 3.0 software (Agilent).

\section{Loss of Heterozygosity Evaluation}

Allelic loss was evaluated according to the peak-height ratio as previously described (23). A high level of $\mathrm{LOH}$ was assumed when the signal intensity of the allele in tumor tissue was less than half of that in the reference DNA (i.e., DNA from the normal brain). $\mathrm{LOH}$ ratio $\leq 0.5$ or $\mathrm{LOH}$ ratio $\geq 1.5$ is defined as a high level of LOH; LOH ratio $>0.5$ or $<1.5$ is defined as low level or no LOH. LOH ratio of $<0.5$ indicates significant loss of allele 2 in the glioma tissue, and a ratio of $>1.5$ indicates the significant loss of allele 1 in the glioma tissue. LOH10q was defined when at least three informative loci were deleted.

$$
\begin{aligned}
\text { LOH ratio }= & \frac{\text { Peak height }(\text { Normal allele } 1)}{\text { Peak height }(\text { Normal allele } 2)} \\
& \div \frac{\text { Peak height }(\text { Glioma allele } 1)}{\text { Peak height }(\text { Glioma allele } 2)}
\end{aligned}
$$

\section{Bioinformatics Analysis}

Gene expression data, DNA copy number information, and survival data were downloaded from cbioportal for cancer genomics (https://www.cbioportal.org/). The Cancer Genome Atlas (TCGA) project used for analyses consisted of clinical and gene expression data from 206 GBM patients (24).

\section{Western Blotting}

Tissue protein lysates were prepared from clinical specimens snap-frozen in liquid nitrogen and stored at $-80^{\circ} \mathrm{C}$ until use. 
Protein lysates were extracted using radioimmunoprecipitation assay (RIPA) lysis buffer with protease inhibitor cocktail. Detailed procedures of immunoblotting were performed as previously described (25). Anti-ADD3 antibody was purchased from Sigma-Aldrich (\#HPA035696); anti-GAPDH antibody (\#2118) was purchased from Cell Signaling Technologies. Protein band intensities were quantified by ImageJ software. Band intensities were measured as the total volume under the peak of interest, with background intensity subtracted under the peak of interest, and normalized to that of the reference protein (GAPDH).

\section{Immunohistochemical Staining}

Immunohistochemical (IHC) staining of formalin-fixed paraffinembedded (FFPE) sections was performed on consecutive $5-\mu \mathrm{m}$ thick sections. Tissue sections were subjected to deparaffinization by xylene and rehydration in serial dilutions of ethanol, followed by heat-induced antigen retrieval in $10 \mathrm{mM}$ of sodium citrate ( $\mathrm{pH}$ 6.0). Endogenous peroxidase was quenched by treatment with $3 \%$ hydrogen peroxide for $30 \mathrm{~min}$, and non-specific protein binding was blocked with 10\% normal goat serum (Dako) for $1 \mathrm{~h}$. Sections were incubated with primary antibodies at appropriate dilutions at $4^{\circ} \mathrm{C}$ overnight in a moist chamber. After incubation, sections were washed with Tris-buffered saline three times, followed by incubation with horseradish peroxidase (HRP)conjugated secondary antibodies (Dako) for $30 \mathrm{~min}$. DAKO EnVision System (Dako) was used to detect signals from DAB chromogen substrate. Finally, sections were counterstained with hematoxylin (Vector Laboratories) and mounted in DPX mounting solution (BDH Laboratory). All IHC sections were quantified and evaluated as the mean value from five random $\times 200$ microscopic fields. All calculations on tissue sections were processed and analyzed by ImageJ software.

\section{Statistical Analysis}

The goals of the statistical analysis were to uncover the clinical association of $10 \mathrm{qLOH}$ by ascertaining molecular genetics and pathologic and clinical parameters. The association between variables was tested with Pearson's chi-squared $\left(\chi^{2}\right)$ test. The Kaplan-Meier method was used to estimate overall survival. The time of an event was calculated as the date of the initial pathological diagnosis until the time to death or the time of the last contact if the patient was alive or the last day of the study period. Differences in survival distributions were evaluated using a log-rank test. All the results were considered statistically significant when the two-sided $p$ was $<0.05$.

\section{RESULTS}

\section{Patient Demographics}

This is a retrospective analysis of 43 glioma cases evaluating the association between allelic loss at chromosome 10q and the clinicopathological features. Table 1 summarizes the patient demographics. In this glioma cohort, 21 were female and 22 were male adults (mean age $53.4 \pm 17.3$ ). There were 8 grade 2 glioma (18.6\%), 12 grade 3 glioma (27.9\%), and 23 grade 4 GBM (53.5\%). According to the anatomical location, the frontal lobe was the most common site involved (22 cases, 51\%). Twelve (28.6\%) patients did not receive temozolomide (TMZ) as concurrent or adjuvant therapy, $7(16.7 \%)$ received less than 6 cycles, and $23(54.7 \%)$ received more than 6 cycles as adjuvant therapy.

\section{Allelic Loss at D10S173 and D10S1137 Is Associated With Tumor Grading and Proliferation}

The associations of allelic loss at each of the $10 \mathrm{q}$ loci examined with clinicopathological features were analyzed by Pearson's $\chi^{2}$ test among the cohort, and the results are summarized in Table 1. LOH at D10S173 and D10S1137 loci demonstrated a significant association with tumor grade $\left({ }^{* *} p<0.001\right.$ and ${ }^{* *} p<$ 0.01 , respectively), as well as the $M I B-1$ proliferative index $\left({ }^{* *} p<\right.$ 0.01 and ${ }^{*} p<0.05$, respectively), with $>10 \%$ MIB-1 being predominant in the high LOH group (78.6\%). Only D10S1483 was associated with $M G M T$ promoter methylation $\left({ }^{*} p<0.05\right)$. We did not observe any correlation with gender, age, tumor location, or TMZ treatment.

\section{Deletion Mapping Revealed Diagnostic Implication of D10S173 in Gliomas}

Five microsatellite markers spanning 10 q23 to 10 q26 were selected for $\mathrm{LOH}$ analyses in 43 cases of gliomas. Figure 1A illustrates the corresponding chromosomal loci and the markers flanking or intragenic of the gene loci. $\mathrm{LOH}$ with three or more loci in all examined chromosomes was considered to amount to the entire loss of the long arm $(55.8 \%$ in all cases, $37.5 \%$ in grade $2,50 \%$ in grade 3 , and $69.5 \%$ in GBM); $18 / 43$ (41.8\%) showed partial or interstitial allelic losses, and only one grade 2 glioma A\#3 was intact. Of the gliomas, 97\% harbor allelic loss in at least one locus. The peak intensity from normal brain tissue $(\mathrm{N \# 1)}$ was used as a reference for $\mathrm{LOH}$ analysis as shown in Figure 1B. It shows the representative peak from capillary electrophoresis, where all the five alleles were lost in one of the grade 3 gliomas $(\mathrm{AA \# 1})$. The frequency of loss in gliomas of different malignancy grades is represented as a deletion map according to their genetic loci (Figure 1C). Among the five microsatellite markers, D10S173 at ADD3/MXI1 locus demonstrated frequent deletion in high-grade gliomas ( $83.3 \%$ in grade 3 and $82.6 \%$ in grade 2 ) and was less common in low-grade cases (12.5\%) (Table 2). The findings suggest the diagnostic potential of allelic deletion, specifically at D10S173, for high-grade gliomas.

\section{Copy Number Deletion of ADD3 and MXI1 at D10S173 Locus Is Associated With Poor Survival}

The clinical outcomes of GBM patients in our cohort were examined using the Kaplan-Meier survival analysis. Log-rank test was calculated for the statistical significance between low $\mathrm{LOH}$ and high $\mathrm{LOH}$ groups. Despite the limitation of the small sample size in our study, statistical significance was achieved in GBM with D10S173 deletion. Patients with high $\mathrm{LOH}$ at 
TABLE 1 | Patient demographics and the association between allelic loss at five chromosomal loci on 10q and the clinicopathological features

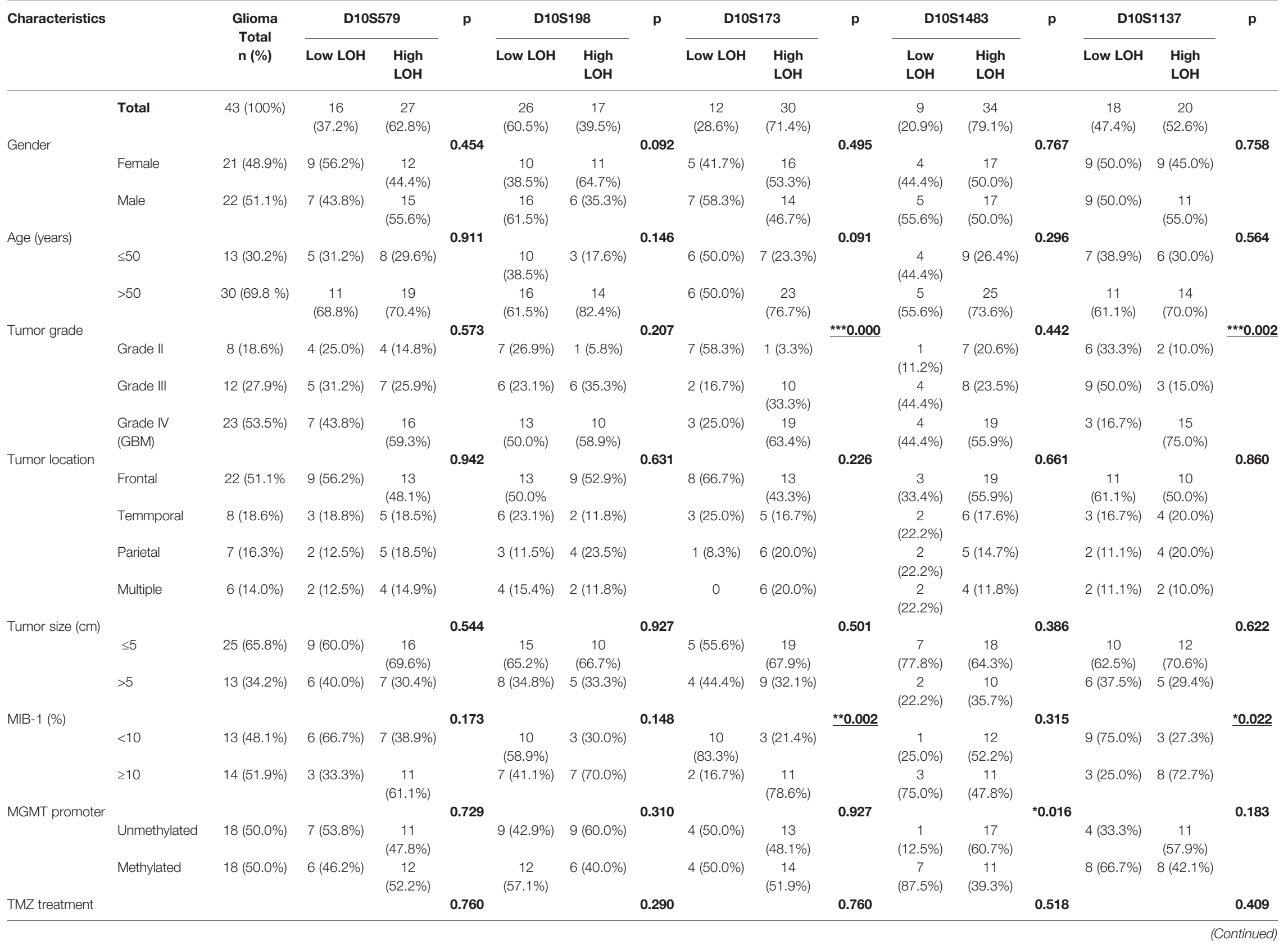




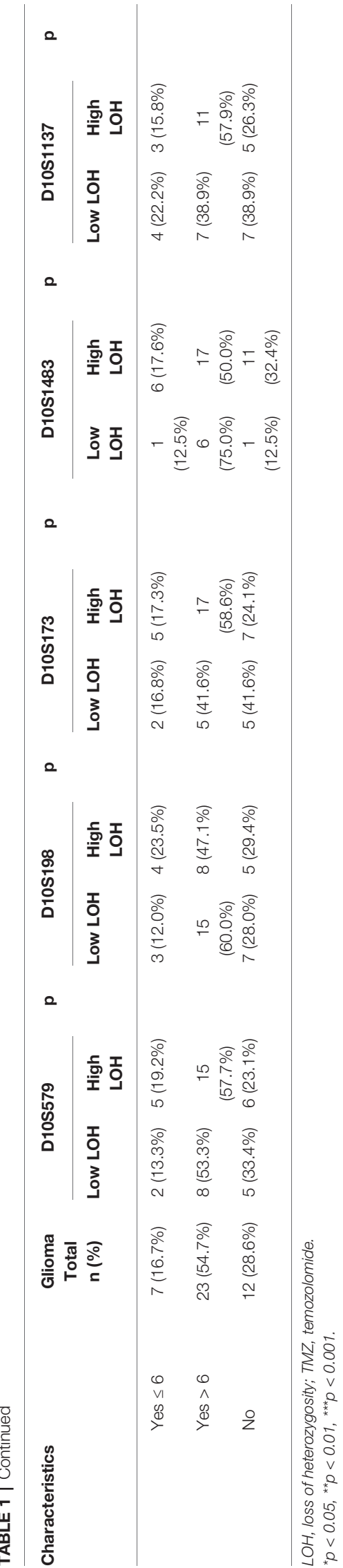

D10S173 had poorer survival than patients with low $\mathrm{LOH}\left({ }^{*} p=\right.$ 0.047) (Figure 2A). Our finding was validated using a larger GBM cohort in the TCGA database, which showed copy number variations of both $A D D 3$ and MXI1 in GBM. Survival analysis of GBM with $A D D 3$ copy number loss, 46 diploid vs. 157 deletion (Figure 2B) and MXI1 copy number loss, and 21 diploid vs. 69 deletion (Figure 2C) showed a significant correlation with an unfavorable outcome (log rank ${ }^{*} p<0.01$ ), with a median survival of 12.95 months in ADD3-deleted tumors compared with 15.65 months in non-deleted (diploid) tumors (*** $p<$ 0.001 ) and median survival of 12.95 months in MXI1 deleted tumors compared with 28.47 months in non-deleted (diploid) tumors $\left.{ }^{* * *} p<0.001\right)$.

\section{ADD3 Loss Is Implicated in Disease Progression}

While the tumor-suppressive role of MXI1 in glioma has been reported, less is known about the role of $A D D 3$ in glioma. In this study, we showed that LOH at D10S173 (ADD3/MXI1) was frequently seen in high-grade gliomas compared with low-grade gliomas and with a high proliferative index, and the results were suggestive of the role of these genes in regulating tumor progression. $A D D 3$ protein expression was determined by Western blotting and IHC in another cohort with 10 patientpaired primary-recurrent gliomas (P1-10). In line with our hypothesis, further losses of $A D D 3$ protein expression were observed in 6 out of 7 gliomas progressing to a higher malignancy grade (P1-P7, Figure 3A). ADD3 was also further downregulated in recurrent GBM compared with its primary lesion (P8-P10) (Figure 3B).

\section{DISCUSSION}

Loss of genetic material at certain chromosomal regions has been considered a major event in tumor development and progression. The assessment of allelic loss is one of the most useful approaches hinting at the loci of potential tumor suppressor genes. Chromosome 10q has been suggested to encompass multiple tumor suppressors apart from PTEN (10q23), on account of the frequent losses observed also at loci in the distal region of $10 \mathrm{q}$ (10q25-qter) $(5,9,10,16)$. In an attempt to assess the possible implications of deletion at different chromosome regions, we examined the frequency of $10 \mathrm{q} \mathrm{LOH}$ in a glioma cohort and evaluated the association with patients' clinicopathological characteristics.

The results from deletion mapping were consistent with those of other studies, where most GBM cases demonstrated a high incidence of allelic loss and appeared to have lost the entire arm of chromosome 10q (69.5\%). Of the five allelic loci examined, only LOH of D10S173 at the ADD3/MXI1 locus was a predictor of shorter survival and had a significant association with tumor grade and proliferative index. MXI1 is a transcription repressor of MYC (26). Despite that somatic mutations were not found in MXI1, which did not seem to support the two-hit hypothesis for gene inactivation as a tumor suppressor, its growth-suppressive 
TABLE 2 | Primer sequences and frequencies of $\mathrm{LOH}$ at five microsatellite markers.

\begin{tabular}{|c|c|c|c|c|c|c|c|}
\hline Microsatellite marker & Location & Size range (bp) & Forward primer $5^{\prime}-3^{\prime}$ & Reverse primer $5^{\prime}-3^{\prime}$ & \multicolumn{3}{|c|}{ LOH } \\
\hline D10S579 & $10 q 23.2$ & $260-276$ & CCGATCAATGAGGAGTGCC & ATACACCCAGCCAATGCTGC & $50.0 \%$ & $58.3 \%$ & $69.6 \%$ \\
\hline D10S173 & $10 q 25.2$ & 155 & GCTGATIITCCTGCTGGTC & TGTTCTGAAGCATTTCCTTG & $12.5 \%$ & $83.3 \%$ & $82.6 \%$ \\
\hline D10S1483 & $10 q 26.13$ & $130-158$ & CAATGCTATCCCGGCTATG & TCAAGACTGCAAGCGTGT & $87.5 \%$ & $66.7 \%$ & $82.6 \%$ \\
\hline D10S1137 & $10 q 26.3$ & 600 & GGAGACAGAGCAAGACCTG & GATGACTCTCCAGCAGCTTC & $33.3 \%$ & $25.0 \%$ & $83.3 \%$ \\
\hline
\end{tabular}

$\mathrm{LOH}$, loss of heterozygosity.
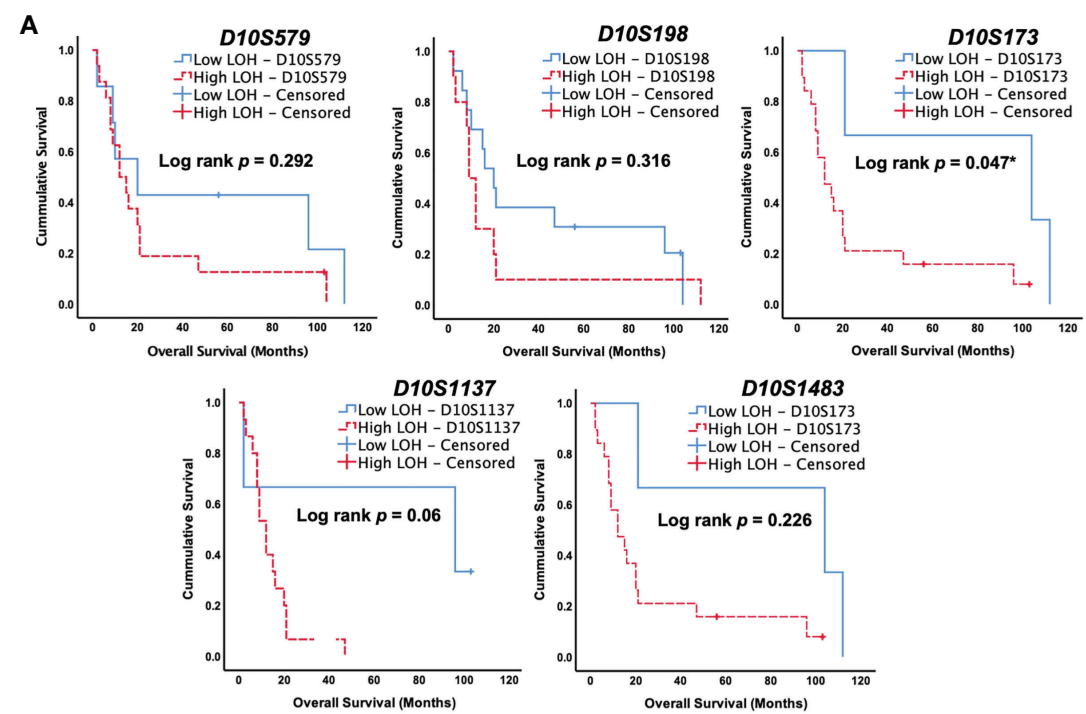

B
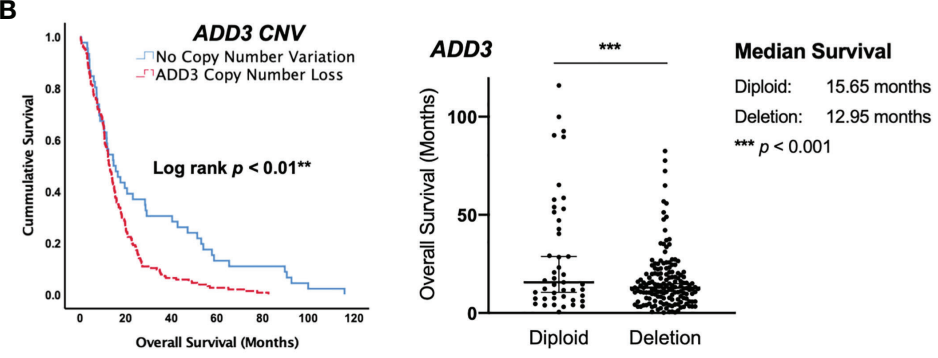

C
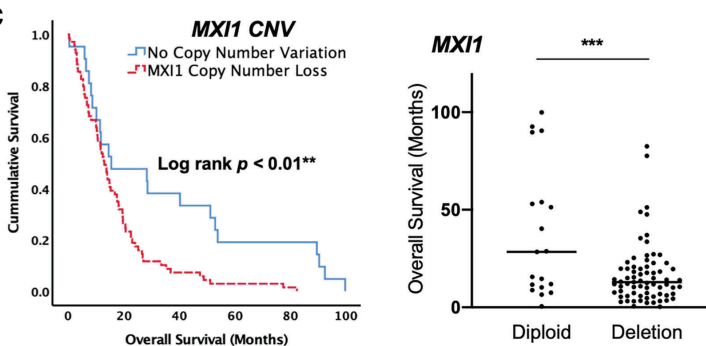

\section{Median Survival}

Diploid: 28.47 months

Deletion: 12.95 months

*** $p<0.001$

FIGURE 2 | Allelic loss at the ADD3 locus is associated with poor survival in glioblastoma (GBM). (A) Kaplan-Meier survival curve in our cohort of GBM. Allelic loss at D10S579, D10S198, D10S1483, and D10S1137 showed no significant difference in patient survival. A high level of deletion at D10S173 was statistically correlated with shorter survival compared with patients that had low-level deletion ( $\left.{ }^{*} p<0.05\right)$. (B, C) The Cancer Genome Atlas (TCGA) database with 203 GBM specimens was used for validation, and data were consistent with those in our cohort, suggesting that a copy number loss of $A D D 3$ and MXI1 (D10S173) is associated with a worse clinical outcome $\left.{ }^{* \star} p<0.01\right)$. ADD3 copy number variation in $202 \mathrm{GBM}$ and the overall patient survival. A total of 157 samples harbor copy number loss, and 45 were diploid. Median with $\left.95 \% \mathrm{Cl}{ }^{* \star \star} \mathrm{p}<0.0001\right)$. MXI1 copy number variation in $90 \mathrm{GBM}$ and the overall patient survival. A total of 71 samples harbor copy number loss, and 19 were diploid. Median with $95 \% \mathrm{Cl}\left({ }^{* \star *} \mathrm{p}<0.0001\right)$. 
A
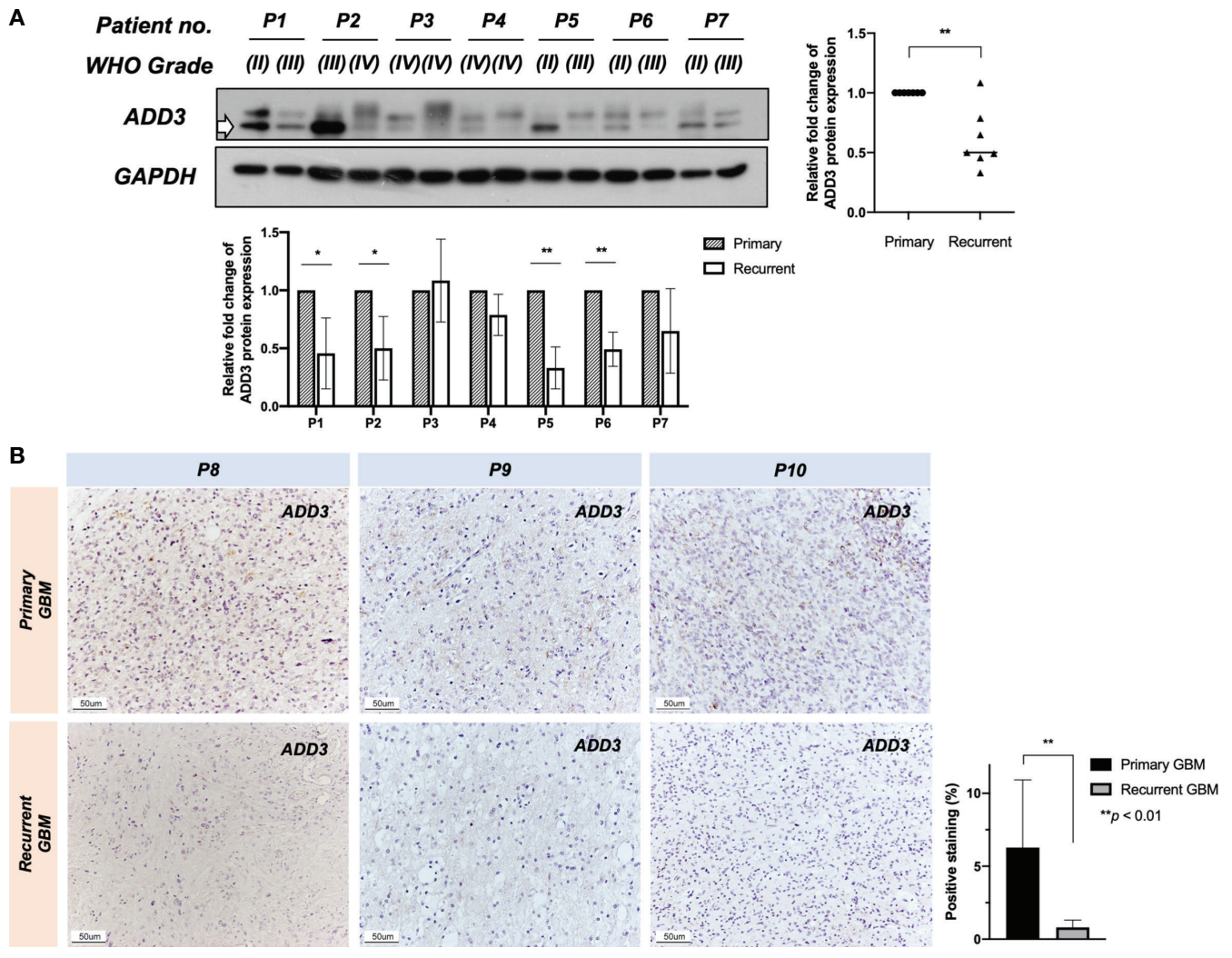

Primary 口 Recurren

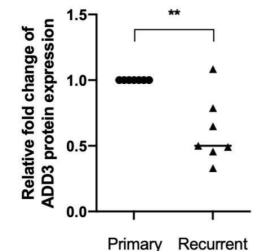

Primary Recurrent

FIGURE 3 | Further loss of ADD3 expression during tumor progression. ADD3 expression was determined in 10 patient-paired primary-recurrent gliomas (P1-P10) with different malignancy grades (WHO grade). (A) Western blotting analysis on seven (P1-P7) recurrent pairs and quantified signal intensity of $A D D 3$ protein bands (bottom band) normalized by GAPDH expression (bottom chart). Six out of seven recurrent gliomas had a further loss of $A D D 3$ expression as compared with its primary tumor (graph on the right). (B) Representative immunohistochemical (IHC) staining of ADD3 in three primary-recurrent glioblastomas (GBMs) (P8-P10) formalin-fixed paraffin-embedded (FFPE) specimens, quantified with staining intensity in three individual tissue specimens for each of the tumors. Original magnification $\times 200$ (scale bar $50 \pm \mu \mathrm{m}$ ). Data represent the mean \pm SD from triplicates $\left({ }^{*} p<0.05,{ }^{* *} p<0.0\right.$ ).

function has been revealed in different cancers $(27,28)$. Compared with MXI1, less is known about the role of $A D D 3$ in cancer. We reported previously on the putative tumorsuppressive function of ADD3 in GBM (22). It is by chance mapped to chromosome 10q25.2, where LOH frequently occurs. $A D D 3$ was found to be significantly downregulated in GBM, and such loss was associated with enhanced tumor growth (22). In this study, we were able to demonstrate the mechanism by which $A D D 3$ was downregulated in gliomas as being most likely due to $\mathrm{LOH}$ with copy number deletions. Our findings further suggested that $A D D 3$ is a putative tumor suppressor in GBM. However, one should note that the current study was conducted before the release of the fifth edition of WHO classification of gliomas published in 2021, which has grouped tumors according to the genetic changes including IDH mutation (29). The term "glioblastoma" has been classified as a specific entity with an IDH wild-type genotype. It is important to note that the term "glioblastoma or GBM" being used in this study is irrespective of the IDH mutation status. Future studies should further refine the prognostic significance of ADD3 in subgroups of tumors with and without IDH mutation.
ADD3 is a crucial assembly factor in the actin cytoskeleton that functions to recruit and promote the formation of spectrin-actin membrane skeleton to provide physical support in the cell $(30,31)$. Given that the cytoskeleton structures are essential for cell motility, downregulation of ADD3 has been shown to be associated with enhanced migratory and invasive potential in lung cancer cells (32). Alteration of ADD3 may also modulate the tumor microenvironment mediated by changes in focal adhesion as well as cell-cell contacts $(32,33)$. It was found that ADD3deficient GBM cells were able to elicit pro-angiogenic signals to stimulate VEGFR expression in endothelial cells (22). All of these could be the potential mechanisms and the pathophysiological consequences that underlie allelic loss of ADD3 in malignant gliomas, particularly during disease progression.

There is accumulating evidence on the prognostic significance of $A D D 3$ in GBM. A recent study by Navarro et al. reported a strong association between EGFR amplification (or EGFRvIII expression) and ADD3 copy number variations in GBM. Genetic clustering suggested the co-occurrence of both events in a subgroup of GBM, the cluster where patients were presented with the shortest survival (34). In accordance with this finding, 
GBMs with EGFR amplification are often accompanied by the complete loss of chromosome 10 and are considered the common phenotypic endpoint of different genetic pathways $(35,36)$. Another notable finding is the progressive event of ADD3 loss during malignant progression in the patient-paired specimens. Indeed, $A D D 3$ deletion appears to occur at a much higher frequency, primarily in the most malignant subtype compared with those in low-grade cases, suggesting that further loss of $A D D 3$ is an event associated with disease progression. In line with this observation, $\mathrm{LOH}$ at $10 \mathrm{q}$-qter was associated with increased proliferative activity, together with the acquisition of a morphological transition to an advanced malignant status (18).

\section{CONCLUSION}

This study provides novel insights into understanding the pathogenesis of malignant progression in glioma. The identification of $A D D 3 / M X I 1$ locus carries diagnostic and prognostic implications and can be particularly helpful for making decisions concerning postoperative management. $A D D 3$ is a putative tumor suppressor that may also serve as a promising marker on $10 \mathrm{q}$ in predicting survival. While a limited number of allelic loci are being tested for LOH10q, pathological tests could be refined with additional markers at loci with higher prognostic significance. The importance of ADD3 gene assumes a new dimension that evaluation on ADD3 expression may be useful to identify patients who are likely to have a progressive disease. The fact that $A D D 3$ is dysregulated at the genetic level should be further explored in regard to the underlying mechanisms of tumor progression and genetic vulnerabilities in glioma. Future studies may target $A D D 3$ depleted tumors as a novel therapeutic approach utilizing synthetic lethality, which can be developed to target gene defects in tumors with $A D D 3$ deletion, similar to the concept of using $P A R P$ inhibitors exclusively for $B R C A$-mutated breast tumors. Future investigations may also focus on the genetic alterations

\section{REFERENCES}

1. Karlbom AE, James CD, Boethius J, Cavenee WK, Collins VP, Nordenskjold M, et al. Loss of Heterozygosity in Malignant Gliomas Involves at Least Three Distinct Regions on Chromosome 10. Hum Genet (1993) 92:169-74. doi: $10.1007 / \mathrm{BF} 00219686$

2. Rasheed BK, McLendon RE, Friedman HS, Friedman AH, Fuchs HE, Bigner DD, et al. Chromosome 10 Deletion Mapping in Human Gliomas: A Common Deletion Region in 10q25. Oncogene (1995) 10:2243-6.

3. Sonoda Y, Murakami Y, Tominaga T, Kayama T, Yoshimoto T, Sekiya T. Deletion Mapping of Chromosome 10 in Human Glioma. Jpn J Cancer Res (1996) 87:363-7. doi: 10.1111/j.1349-7006.1996.tb00231.x

4. Voesten AM, Bijleveld EH, Westerveld A, Hulsebos TJ. Fine Mapping of a Region of Common Deletion on Chromosome Arm 10p in Human Glioma. Genes Chromosomes Cancer (1997) 20:167-72. doi: 10.1002/(SICI)1098-2264 (199710)20:2<167::AID-GCC7>3.0.CO;2-1

5. Ichimura K, Schmidt EE, Miyakawa A, Goike HM, Collins VP. Distinct Patterns of Deletion on 10p and 10q Suggest Involvement of Multiple Tumor Suppressor Genes in the Development of Astrocytic Gliomas of Different Malignancy Grades. Genes Chromosomes Cancer (1998) 22:9-15. doi: 10.1002/(SICI)1098-2264(199805)22:1<9::AID-GCC2>3.0.CO;2-1 associated with $A D D 3$ and the possible regulatory mechanisms on the transcriptional and post-transcriptional levels.

\section{DATA AVAILABILITY STATEMENT}

The raw data supporting the conclusions of this article will be made available by the authors, without undue reservation.

\section{ETHICS STATEMENT}

The studies involving human participants were reviewed and approved by the Institution Review Board of the University of Hong Kong/Hospital Authority Hong Kong West Cluster. Written informed consent to participate in this study was provided by the participants' legal guardian/next of kin.

\section{AUTHOR CONTRIBUTIONS}

KK carried out the experiments and wrote the paper. SS performed the statistical analysis and collected the data. GL conceived the study and contributed to the critical revision. All authors contributed to the article and approved the submitted version.

\section{ACKNOWLEDGMENTS}

The authors would like to acknowledge the Faculty Core Facility in our institute for their excellent technical assistance in DNA fragment analysis. The contents of this manuscript were partly presented in a poster session at the annual meeting of Hong Kong Neurosurgical Society in 2020 and the American Association for Cancer Research in 2021.

6. Ohgaki H, Dessen P, Jourde B, Horstmann S, Nishikawa T, Di Patre PL, et al. Genetic Pathways to Glioblastoma: A Population-Based Study. Cancer Res (2004) 64:6892-9. doi: 10.1158/0008-5472.CAN-04-1337

7. van den Bent MJ, Weller M, Wen PY, Kros JM, Aldape K, Chang S. A Clinical Perspective on the 2016 WHO Brain Tumor Classification and Routine Molecular Diagnostics. Neuro Oncol (2017) 19:614-24. doi: 10.1093/neuonc/now277

8. Kakkar A, Suri V, Jha P, Srivastava A, Sharma V, Pathak P, et al. Loss of Heterozygosity on Chromosome $10 \mathrm{q}$ in Glioblastomas, and Its Association With Other Genetic Alterations and Survival in Indian Patients. Neurol India (2011) 59:254-61. doi: 10.4103/0028-3886.79139

9. Lin H, Bondy ML, Langford LA, Hess KR, Delclos GL, Wu X, et al. Allelic Deletion Analyses of MMAC/PTEN and DMBT1 Loci in Gliomas: Relationship to Prognostic Significance. Clin Cancer Res (1998) 4:2447-54.

10. Jesionek-Kupnicka D, Szybka M, Potemski P, Kulczycka-Wojdala D, Jaskolski D, Bienkowski M, et al. Association of Loss of Heterozygosity With Shorter Survival in Primary Glioblastoma Patients. Pol J Pathol (2013) 64:268-75. doi: 10.5114/pjp.2013.39335

11. Gasparotto D, Vukosavljevic T, Piccinin S, Barzan L, Sulfaro S, Armellin M, et al. Loss of Heterozygosity at 10q in Tumors of the Upper Respiratory Tract Is Associated With Poor Prognosis. Int J Cancer (1999) 84:432-6. doi: 10.1002/(SICI)1097-0215(19990820)84:4<432::AID-IJC18>3.0.CO;2-\# 
12. Long J, Zhang ZB, Liu Z, Xu YH, Ge CL. Loss of Heterozygosity at the Calcium Regulation Gene Locus on Chromosome 10q in Human Pancreatic Cancer. Asian Pac J Cancer Prev (2015) 16:2489-93. doi: 10.7314/APJCP.2015.16.6.2489

13. Petersen S, Wolf G, Bockmuhl U, Gellert K, Dietel M, Petersen I. Allelic Loss on Chromosome 10q in Human Lung Cancer: Association With Tumour Progression and Metastatic Phenotype. Br J Cancer (1998) 77:270-6. doi: 10.1038/bjc.1998.43

14. Thiagalingam S, Laken S, Willson JK, Markowitz SD, Kinzler KW, Vogelstein B, et al. Mechanisms Underlying Losses of Heterozygosity in Human Colorectal Cancers. Proc Natl Acad Sci USA (2001) 98:2698-702. doi: 10.1073/pnas.051625398

15. Scott DK, Straughton D, Cole M, Bailey S, Ellison DW, Clifford SC. Identification and Analysis of Tumor Suppressor Loci at Chromosome 10q23.3-10q25.3 in Medulloblastoma. Cell Cycle (2006) 5:2381-9. doi: 10.4161/cc.5.20.3360

16. Albarosa R, Colombo BM, Roz L, Magnani I, Pollo B, Cirenei N, et al. Deletion Mapping of Gliomas Suggest the Presence of Two Small Regions for Candidate Tumor-Suppressor Genes in a 17-cM Interval on Chromosome 10q. Am J Hum Genet (1996) 58:1260-7.

17. Fults D, Pedone CA, Thompson GE, Uchiyama CM, Gumpper KL, Iliev D, et al. Microsatellite Deletion Mapping on Chromosome $10 \mathrm{q}$ and Mutation Analysis of MMAC1, FAS, and MXI1 in Human Glioblastoma Multiforme. Int J Oncol (1998) 12:905-10. doi: 10.3892/ijo.12.4.905

18. Fujisawa H, Kurrer M, Reis RM, Yonekawa Y, Kleihues P, Ohgaki H. Acquisition of the Glioblastoma Phenotype During Astrocytoma Progression Is Associated With Loss of Heterozygosity on 10q25-Qter. Am J Pathol (1999) 155:387-94. doi: 10.1016/S0002-9440(10)65135-8

19. van den Boom J, Wolter M, Kuick R, Misek DE, Youkilis AS, Wechsler DS, et al. Characterization of Gene Expression Profiles Associated With Glioma Progression Using Oligonucleotide-Based Microarray Analysis and Real-Time Reverse Transcription-Polymerase Chain Reaction. Am J Pathol (2003) 163:1033-43. doi: 10.1016/S0002-9440(10)63463-3

20. Huang H, Colella S, Kurrer M, Yonekawa Y, Kleihues P, Ohgaki H. Gene Expression Profiling of Low-Grade Diffuse Astrocytomas by cDNA Arrays. Cancer Res (2000) 60:6868-74.

21. Mariani L, Beaudry C, McDonough WS, Hoelzinger DB, Demuth T, Ross KR, et al. Glioma Cell Motility Is Associated With Reduced Transcription of Proapoptotic and Proliferation Genes: A cDNA Microarray Analysis. J Neurooncol (2001) 53:161-76. doi: 10.1023/A:1012253317934

22. Kiang KM, Zhang P, Li N, Zhu Z, Jin L, Leung GK. Loss of Cytoskeleton Protein ADD3 Promotes Tumor Growth and Angiogenesis in Glioblastoma Multiforme. Cancer Lett (2020) 474:118-26. doi: 10.1016/j.canlet.2020.01.007

23. Ramos TD, Amorim LM. Moleuclar Biology Techniques for Loss of Heterozygosity Detection: The Glioma Example. J Bras Patol Med Lab (2015) 51:189-96. doi: 10.5935/1676-2444.20150033

24. N. Cancer Genome Atlas Research. Comprehensive Genomic Characterization Defines Human Glioblastoma Genes and Core Pathways. Nature (2008) 455:1061-8. doi: 10.1038/nature07385

25. Sun S, Kiang KMY, Ho ASW, Lee D, Poon MW, Xu FF, et al. Endoplasmic Reticulum Chaperone Prolyl 4-Hydroxylase, Beta Polypeptide (P4HB) Promotes Malignant Phenotypes in Glioma via MAPK Signaling. Oncotarget (2017) 8:71911-23. doi: 10.18632/oncotarget.18026

26. Wechsler DS, Shelly CA, Petroff CA, Dang CV. MXI1, a Putative Tumor Suppressor Gene, Suppresses Growth of Human Glioblastoma Cells. Cancer Res (1997) 57:4905-12.
27. Prochownik EV, Eagle Grove L, Deubler D, Zhu XL, Stephenson RA, Rohr LR, et al. Commonly Occurring Loss and Mutation of the MXI1 Gene in Prostate Cancer. Genes Chromosomes Cancer (1998) 22:295-304. doi: 10.1002/(SICI) 1098-2264(199808)22:4<295::AID-GCC5>3.0.CO;2-Q

28. Tsao CC, Teh BT, Jonasch E, Shreiber-Agus N, Efstathiou E, Hoang A, et al. Inhibition of Mxil Suppresses HIF-2alpha-Dependent Renal Cancer Tumorigenesis. Cancer Biol Ther (2008) 7:1619-27. doi: 10.4161/cbt.7.10.6583

29. Louis DN, Perry A, Wesseling P, Brat DJ, Cree IA, Figarella-Branger D, et al. The 2021 WHO Classification of Tumors of the Central Nervous System: A Summary. Neuro Oncol (2021) 23:1231-51. doi: 10.1093/neuonc/noab106

30. Gardner K, Bennett V. Modulation of Spectrin-Actin Assembly by Erythrocyte Adducin. Nature (1987) 328:359-62. doi: 10.1038/328359a0

31. Bennett V, Gardner K, Steiner JP. Brain Adducin: A Protein Kinase C Substrate That may Mediate Site-Directed Assembly at the Spectrin-Actin Junction. J Biol Chem (1988) 263:5860-9. doi: 10.1016/S0021-9258(18)60645-3

32. Lechuga S, Amin PH, Wolen AR, Ivanov AI. Adducins Inhibit Lung Cancer Cell Migration Through Mechanisms Involving Regulation of Cell-Matrix Adhesion and Cadherin-11 Expression. Biochim Biophys Acta Mol Cell Res (2019) 1866:395-408. doi: 10.1016/j.bbamcr.2018.10.001

33. Naydenov NG, Ivanov AI. Adducins Regulate Remodeling of Apical Junctions in Human Epithelial Cells. Mol Biol Cell (2010) 21:3506-17. doi: 10.1091/ mbc.e10-03-0259

34. Navarro L, San-Miguel T, Megias J, Santonja N, Calabuig S, Munoz-Hidalgo L, et al. Identification of New Genetic Clusters in Glioblastoma Multiforme: EGFR Status and ADD3 Losses Influence Prognosis. Cells (2020) 9:2429. doi: $10.3390 /$ cells9112429

35. Fujisawa H, Reis RM, Nakamura M, Colella S, Yonekawa Y, Kleihues P, et al. Loss of Heterozygosity on Chromosome 10 Is More Extensive in Primary (De Novo) Than in Secondary Glioblastomas. Lab Invest (2000) 80:65-72. doi: 10.1038/labinvest.3780009

36. Leenstra S, Oskam NT, Bijleveld EH, Bosch DA, Troost D, Hulsebos TJ Genetic Sub-Types of Human Malignant Astrocytoma Correlate With Survival. Int J Cancer (1998) 79:159-65. doi: 10.1002/(SICI)1097-0215 (19980417)79:2<159::AID-IJC11>3.0.CO;2-5

Conflict of Interest: The authors declare that the research was conducted in the absence of any commercial or financial relationships that could be construed as a potential conflict of interest.

Publisher's Note: All claims expressed in this article are solely those of the authors and do not necessarily represent those of their affiliated organizations, or those of the publisher, the editors and the reviewers. Any product that may be evaluated in this article, or claim that may be made by its manufacturer, is not guaranteed or endorsed by the publisher.

Copyright (c) 2021 Kiang, Sun and Leung. This is an open-access article distributed under the terms of the Creative Commons Attribution License (CC BY). The use, distribution or reproduction in other forums is permitted, provided the original author(s) and the copyright owner(s) are credited and that the original publication in this journal is cited, in accordance with accepted academic practice. No use, distribution or reproduction is permitted which does not comply with these terms. 